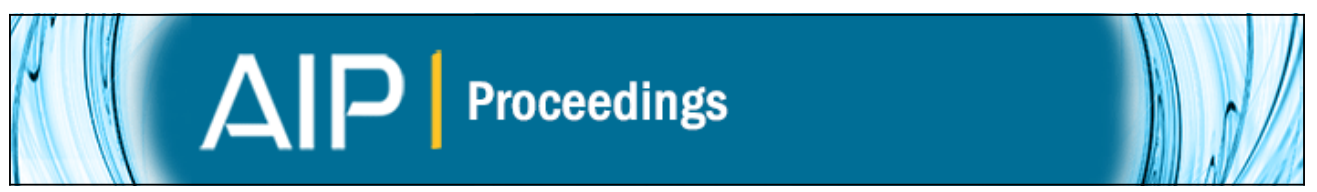

\title{
Quantal Time Asymmetry: Mathematical Foundation and Physical Interpretation
}
A. Bohm

Citation: AIP Conference Proceedings 1259, 157 (2010); doi: 10.1063/1.3479296

View online: http://dx.doi.org/10.1063/1.3479296

View Table of Contents:

http://scitation.aip.org/content/aip/proceeding/aipcp/1259?ver=pdfcov

Published by the AIP Publishing

\section{Articles you may be interested in}

The Everett-Wheeler interpretation and the open future

AIP Conf. Proc. 1327, 492 (2011); 10.1063/1.3567480

Entropic Time

AIP Conf. Proc. 1305, 200 (2011); 10.1063/1.3573617

Interpretation of Quantum Mechanics. A view of our universe

AIP Conf. Proc. 1177, 10 (2009); 10.1063/1.3253958

On Mathematical Modeling Of Quantum Systems

AIP Conf. Proc. 1146, 105 (2009); 10.1063/1.3183535

Statistical quantum probabilities in coherent bosonic and fermionic states

J. Math. Phys. 47, 083302 (2006); 10.1063/1.2259583 


\title{
Quantal Time Asymmetry: Mathematical Foundation and Physical Interpretation
}

\author{
A. Bohm \\ Department of Physics, University of Texas at Austin, Austin, TX 78712, U.S.A.
}

\begin{abstract}
Time in standard quantum mechanics extends from $-\infty<t<+\infty$; this is the result of a mathematical theorem (Stone-von Neumann) for the solutions of the Schrödinger equation for states or of the Heisenberg equation for observables. In reality $t$ does not extend to $t \rightarrow-\infty$, since according to causality, a quantum state $\phi^{+}$must be prepared first at a particular time $t=t_{0}$, before the probability $\left|\left(\psi^{-}(t), \phi^{+}\left(t_{0}\right)\right)\right|^{2}$ for an observable $\psi^{-}$can be measured in it at $t>t_{0}$ (Feynman (1948)). In experiments on single $\mathrm{Ba}^{+}$ions, Dehmelt and others observed this finite preparation time as the ensemble of onset-times $t_{0}^{1}, t_{0}^{2}, \ldots, t_{0}^{n}$ of dark periods. How the semigroup time evolution, $t_{0} \equiv 0<t<\infty$ with a beginning of time $t_{0}$, can suggest the parametrization of the resonance pole position of the $Z$-boson at $s=s_{R}$ as $s_{R}=\left(M_{R}-i \Gamma_{R} / 2\right)^{2}$ in terms of a mass $M_{R}$ and a width $\Gamma_{R}$ given by a lifetime $\tau=\hbar / \Gamma_{R}$, is the subject of this contribution dedicated to Augusto García.
\end{abstract}

Keywords: rigged Hilbert space, resonances, $Z$ boson, time asymmetry

PACS: 03.65.Db, 03.65.Nk, 11.10.St, 14.70.Hp

\section{INTRODUCTION}

The definition of mass and width of a relativistic resonance has been a problem for a long time. For some time the Particle Data Group [1] listed two or three different values for the mass of the $Z$-boson and some of the hadron resonances obtained from the same experimental data with different (theoretical) definitions for the lineshape of a resonance. It was Augusto García who told me, during one of my visits at CINVESTAV ${ }^{1}$, that the standard definition $\left(M_{Z}, \Gamma_{Z}\right)$ for the $Z$-boson, based on the on-mass-shell renormalization scheme, is not gauge invariant (NNLO, $\mathscr{O}\left(g^{6}\right)$ and $\mathscr{O}\left(g^{4}\right)$ ) [2]. This started me on the problem of the definition of relativistic resonances.

There are two different definitions of the $Z$-boson lineshape For the propagator definition in the For the $S$-matrix pole definition: on-mass-shell-renormalization scheme:

$$
a_{j}^{\text {res }}=\frac{R_{Z}}{s-M_{Z}^{2}+i \frac{s}{M_{Z}} \Gamma_{Z}}
$$

$$
a_{j}^{\mathrm{res}}=\frac{r}{s-s_{R}}
$$

\footnotetext{
${ }^{1}$ Centro de Investigación y de Estudios Avanzados del Instituto Politécnico Nacional, Mexico
} 
Here $s_{R}$ is the pole position of the complex $S$-matrix pole, and

$$
\begin{aligned}
a_{j}^{\mathrm{res}} & =\frac{r}{s-\bar{M}_{Z}^{2}+i \bar{M}_{Z} \bar{\Gamma}_{Z}} \\
a_{j}^{\mathrm{res}} & =\frac{r}{s-\left(M_{R}-i \Gamma_{R} / 2\right)^{2}}
\end{aligned}
$$

are two different parametrizations of the pole position $s_{R}$.

Different "experimental" values of $(M, \Gamma)$ are obtained from fits of the partial wave amplitude of angular momentum $j$,

$$
a_{j}(s)=a_{j}^{\mathrm{res}}(s)+B_{j}(s)
$$

to the cross section data ("line shape") $\left|a_{j}(s)\right|^{2}$ (and also to the asymmetries) ${ }^{2}$.

The different parametrizations of the resonance amplitude (1), (2), (3) lead to different "experimental" values of the resonance mass and width ${ }^{3}$ :

$$
\begin{aligned}
M_{Z} & =91.1875 \pm 0.0021 \mathrm{GeV} & \Gamma_{Z} & =2.4939 \pm 0.0024 \mathrm{GeV} \\
M_{R} & =91.1611 \pm 0.0023 \mathrm{GeV} & \Gamma_{R} & =2.4943 \pm 0.0024 \mathrm{GeV} \\
\bar{M}_{Z} & =91.1526 \pm 0.0023 \mathrm{GeV} & \bar{\Gamma}_{Z} & =2.4945 \pm 0.0024 \mathrm{GeV} \\
M_{Z}-M_{R} & =0.026 \pm 0.004 \mathrm{GeV} & M_{Z}-\bar{M}_{Z} & =0.035 \pm 0.004 \mathrm{GeV}
\end{aligned}
$$

This differences between the different mass values are significant as compared to the experimental errors $\sigma_{M_{Z}}=0.0021 \mathrm{GeV}$, and therefore one may ask the question: Which of these $(M, \Gamma)$ is the right mass and width?

The same situation also holds for the different mass definitions of the hadron resonances for which sufficiently accurate data are available, discussed extensively at CINVESTAV [4]

$$
M_{\Delta}-\bar{M}_{\Delta} \approx 19 \mathrm{MeV} \quad\left(\sim 2 \% \text { of } M_{\Delta}\right) \quad M_{\rho}-\bar{M}_{\rho} \approx 10 \mathrm{MeV} \quad\left(\sim 1.5 \% \text { of } M_{\rho}\right)
$$

Thus one has again the same important question: What is the right definition of mass $M$ and width $\Gamma$ ?

This leads to more general questions: What is the a resonance? Does a resonance have a lifetime even if one cannot measure it? In non-relativistic (atomic, molecular) physics, the majority opinion is: a resonance of width $\Gamma$ and a decaying state of lifetime $\tau$ are just different manifestations of the same state and $\tau=\hbar / \Gamma$. The opposite opinion dominates in relativistic particle physics: a lifetime $\tau$ for broad relativistic resonances is not defined.

In these lecture notes we want to address a more positive question: can the relation $\tau=\hbar / \Gamma$ between an experimental lifetime $\tau$ of a decaying state and a width $\Gamma$ of

\footnotetext{
2 The amplitude $B_{j}(s)$ is slowly varying near the singularity of $(1) ; B_{j}(s)$ describes the non-resonant background (or contribution from other, far away, resonances.

${ }^{3} M_{Z}, \bar{M}_{Z}$ and $M_{R}$ were listed in PDG book [1], now only $\left(M_{Z}, \Gamma_{Z}\right)[3]$ is listed as the standard value.
} 
the resonance help us to find the right definition for mass and width of a relativistic resonance? Which of the parametrizations used in (1), (2), (3) or any other thinkable parametrizations fulfills the relation $\tau=\hbar / \Gamma$ ? The answer to this question would unify the theory of relativistic resonances and decaying states.

The starting point for a relativistic theory of particles and fields [5] is Wigner's definition of stable relativistic particles by unitary irreducible representations $(m, j)$ of the Poincare group (the invariance group of relativistic space-time). In analogy to this definition of stable relativistic particles by the unitary representations ( $m=$ real mass, $j=$ integer, or half integer spin), a quasistable relativistic particle should be defined by a semigroup representation of the Poincaré group. The semigroup representation is characterized by $\left(s_{R} \equiv M_{R}-i \Gamma_{R} / 2, j\right)$; here $j$ is again spin and $s_{R}$ is the position of the resonance pole of the $j$-th partial $S$-matrix element $S_{j}^{\eta^{\prime} \eta}(s)$, where $s=p_{\mu} p^{\mu}$. The invariant $s$ can be analytically continued into the complex plane second sheet, and $\eta^{\prime} \eta$ are the particle species numbers [5] or channel quantum numbers of the in- and outconfiguration. The two parameters $(M, \Gamma)$ or $(M, \hbar / \tau)$ are also the two parameters by which one catalogs the relativistic resonances or the decaying states in [1]. However in the relativistic case it is not quite clear how one should divide the $j$-th partial wave amplitude (4) into an amplitude specific to the resonance $a_{j}^{\text {res }}(s)$ and the rest $B_{j}(s)$. This is the problem discussed in these lecture notes.

\section{RESONANCES AND DECAYING STATES}

For the sake of simplicity, and since the talk was addressed to a broader audience we want to discuss here mainly the non-relativistic case. We show how a physically minor but mathematically significant modification of quantum theory leads to $\tau=\hbar / \Gamma$ as an exact equality for the non-relativistic case, and then also for the relativistic case.

\section{Comparing}

\section{Resonances}

Resonances characterized by $\left(E_{R}, \Gamma\right)$ appear as Breit-Wigner lineshape in the cross section

$$
\begin{aligned}
& \sigma_{j}(E) \sim\left|a_{j}(E)\right|^{2} \\
& =\left|\frac{r_{\eta}}{E-\left(E_{R}-i \frac{\Gamma}{2}\right)}+B(E)\right|^{2}
\end{aligned}
$$

$B(E)$ is a slowly varying function of $E$ (background).

\section{and Decaying States}

Decaying states characterized by $\left(E_{D}, \tau\right)$, are measured by the exponential law for the counting rate of the decay products $\eta$

$$
\frac{\Delta N\left(t_{i}\right)}{\Delta t_{i}}=R_{\eta}(t) \propto \mathrm{e}^{-\frac{t}{\tau}},
$$

here $\Delta N\left(t_{i}\right)$ is the number of decay products registered in the detector during the time interval $\Delta t_{i}$ around $t_{i} . t_{i}$ is the lifetime of each individual decaying particle created at $t=0\left(=t_{0}\right)$ 
The fit of the $Z$-boson resonance data with $\sqrt{s}=E$ exhibits the Breit-Wigner

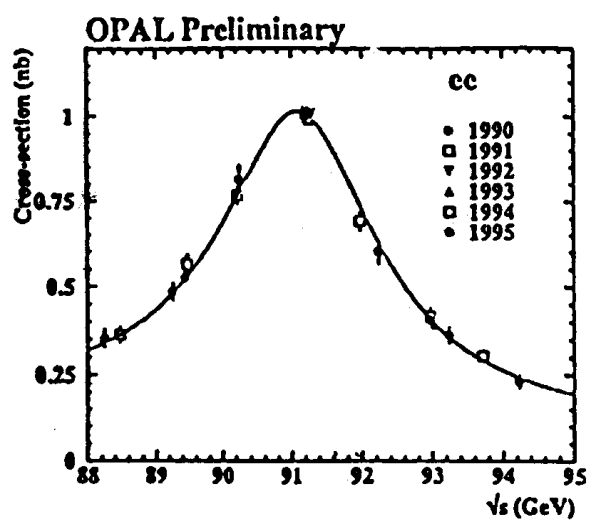

FIGURE 1.
For the $K_{S}^{0}$ decay rate (with the $K_{L}^{0}$ interference analyzed out [6]) the time dependence is exponential

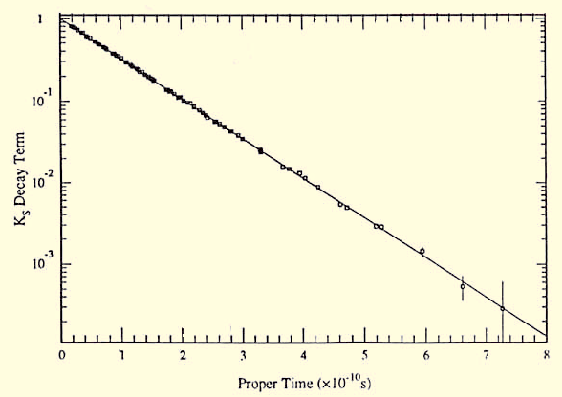

FIGURE 2.

Many people think that resonances are the same as decaying states, and especially for non-relativistic quantum mechanics, a common assumption is that

$$
\frac{\hbar}{\Gamma}=\tau, \quad\left(\text { or at least } \frac{\hbar}{\Gamma} \approx \tau\right) .
$$

This relation is based on the Weisskopf-Wigner (W-W) approximation [7].

But in standard quantum theory there is no proof of $\hbar / \Gamma=\tau$. Using W-W methods, the probability to observe the decay products $|\psi\rangle\langle\psi|$ in a prepared resonance state $\phi(t)$ with Breit-Wigner width $\Gamma$ is derived as [8]

$$
|\langle\psi \mid \phi(t)\rangle|^{2}=\mathscr{P}_{\phi(t)}(\psi) \sim \mathrm{e}^{-\Gamma t / \hbar}+\Gamma \times(\text { additional terms }) .
$$

One can prove that there is no Hilbert space vector $\phi(t)$ that obeys the exact exponential law, i.e., for which (additional terms of (8)) $=0$.

In Fig. 2 for the $K_{S}^{0}$-decay experiment (with $K_{L}^{0}$ interference analyzed out [6]) the time dependence of the decay rate looks perfectly exponential. This means the calculated Born probability for detecting $\pi \pi$ in $K_{S}^{0}$ decay

$$
\left|\left\langle\psi_{\pi \pi} \mid \phi^{K_{S}^{0}}(t)\right\rangle\right|^{2}=\frac{N(t)}{N},
$$

needs to be a perfect exponential, as shown for $N(t)$ by the straight line in Fig. 2 .

The easiest way to derive an exactly exponential decay probability is to postulate a state vector $\phi^{G}$ which has the property:

$$
H \phi^{G}=\left(E_{R}-i \frac{\Gamma}{2}\right) \phi^{G} \quad \text { (10a) } \quad \text { and } \quad \phi^{G}(t)=\mathrm{e}^{-i H t / \hbar} \phi^{G},
$$

The decay probability into any observable $|\psi\rangle\langle\psi|$ is then obtained by using (10b) and 
then (10a) in the exponent:

$$
\begin{aligned}
\mathscr{P}_{\phi^{G}(t)}(\psi)=\left|\left\langle\psi \mid \phi^{G}(t)\right\rangle\right|^{2}= & \left|\left\langle\psi\left|\mathrm{e}^{-i H t / \hbar}\right| \phi^{G}(t)\right\rangle\right|^{2} \\
& =\left|\left\langle\psi \mid \phi^{G}\right\rangle \mathrm{e}^{-i\left(E_{R}-i \Gamma / 2\right) t / \hbar}\right|^{2}=\left|\left\langle\psi \mid \phi^{G}\right\rangle\right|^{2} \mathrm{e}^{-\Gamma t / \hbar} .
\end{aligned}
$$

Though this leads to the exponential law (11), this vector $\phi^{G}$ (called Gamow vector [9]) makes no sense in standard (Hilbert space) quantum mechanics. The reason is that if one solves the Dynamical Equations:

either in the Heisenberg picture

for observables $\Lambda(t)$ or $|\psi(t)\rangle\langle\psi(t)|$

$$
\begin{aligned}
i \hbar \frac{\partial \Lambda(t)}{\partial t} & =-[H, \Lambda(t)] \\
i \hbar \frac{\partial}{\partial t} \psi(t) & =-H \psi(t)
\end{aligned}
$$

or in the Schrödinger picture for states $\phi(t)$

$$
i \hbar \frac{\partial}{\partial t} \phi(t)=H \phi(t)
$$

under standard quantum mechanical boundary conditions:

$$
\text { set of states }\{\phi\}=\mathscr{H}=\text { Hilbert space }=\text { set of observables }\{\psi\}
$$

or

$$
\phi(E)=\langle E \mid \phi\rangle \in L^{2}(E) \quad \psi(E)=\langle E \mid \psi\rangle \in L^{2}(E), \quad E_{0}<E<\infty,
$$

then one obtains (by the Stone-von Neumann Theorem) the unitary group evolution:

$$
\begin{array}{ccc}
\psi(t)=\mathrm{e}^{i H t / \hbar} \psi, & \phi(t)=\mathrm{e}^{-i H t / \hbar} \phi \\
-\infty<t<+\infty & \text { or } & -\infty<t<+\infty \\
\text { (in the Heisenberg picture) } & & \text { (in the Schrödinger picture). }
\end{array}
$$

This would lead to the "exponential catastrophe" for a Gamow vector

$$
\mathscr{P}_{\phi^{G}(t)}(\psi) \sim \mathrm{e}^{-\Gamma t / \hbar} \rightarrow \infty \quad \text { if one extends } t \text { to }-\infty,
$$

as dictated by the Stone-von Neumann theorem in (14).

To give a mathematical meaning to a Gamow vector, one needs another theory than the one based on axiom (13). This theory must:

1. extend the energy to discrete and continuous values in the complex energy plane

2. restrict the time to $0 \equiv t_{0} \leq t<+\infty$ because causality requires that:

(a) a $K_{S}^{0}$ must be prepared first at a time $t=t_{0}$ before one can detect its decay products $\pi^{+} \pi^{-}$at $t>t_{0}$.

(b) it avoid the exponential catastrophe (15).

We have become so used to time $t$ extending over $-\infty<t<+\infty$ in (14),

$$
U(t)=\mathrm{e}^{i H t / \hbar} ; \quad-\infty<t<+\infty,
$$

that we forget that the unitary group (16) is a consequence of a mathematical theorem, the Stone-von Neumann theorem for the Hilbert space (i.e., Lebesgue square integrable 
energy wave function $\phi(E)$ ). The Hilbert space of Lebesgue square integrable functions [10] was a great achievement for the development of a mathematical theory for quantum mechanics. But as documented by the recent textbooks of quantum mechanics, physicists do not use Lebesgue integration. They simply follow the Dirac formulation which is mathematically justified by the Schwartz space. In addition physicists continued the energy of the Dirac kets into the complex energy plane using Lippmann-Schwinger kets. But they hesitated to accept the Gamow kets like (10a) because $-\infty<t<+\infty$ in (14) and (16) leads to the "catastrophe" (15).

The restriction $t_{0}<t<+\infty$ is more natural than $-\infty<t<+\infty$. The finite $t_{0}$ can be given the meaning of the time at which the state $\phi^{K_{S}^{0}}\left(t_{0}\right)$ in (9) is prepared. And a state $\phi^{K_{S}^{0}}\left(t_{0}\right)$ needs to be prepared first before the observable $\left|\psi_{\pi \pi}(t)\right\rangle\left\langle\psi_{\pi \pi}(t)\right|$ can be detected in this state for $t>t_{0}$. Physically $N(t) / N$ of (9) can be counted only for times $t_{i}>t_{0}$, and thus its theoretical representative $\left|\left\langle\phi^{K_{S}^{0}}\left(t_{0}\right) \mid \psi_{\pi \pi}(t)\right\rangle\right|^{2}$, needs to exist for $t>t_{0}$ only.

\section{QUANTUM MECHANICAL TIME ASYMMETRY Quantum Mechanical "Beginning of Time"}

From the remarks at the end of the previous section the experimental situation is much better described by a time evolution semi-group $\mathscr{U}(t)$ with $t_{0}=0 \leq t<\infty$ than by the unitary group $U(t)$ of (16) and (14), because an obvious requirement of causality is that:

A state $\phi$ must be prepared before the observable $|\psi(t)\rangle\langle\psi(t)|$ can be measured in it. The detector cannot count the decay products before the decaying state has been prepared.

This means we have a Quantum Mechanical Arrow of Time:

The Born probability to measure the observable $|\psi(t)\rangle\langle\psi(t)|$ in the state $\phi$

$$
\mathscr{P}_{\phi}(\psi(t))=|\langle\psi(t) \mid \phi\rangle|^{2}=\left|\left\langle e^{i H t} \psi \mid \phi\right\rangle\right|^{2}=\left|\left\langle\psi \mid e^{-i H t} \phi\right\rangle\right|^{2}=|\langle\psi \mid \phi(t)\rangle|^{2}
$$

exists (experimentally) only for $t \geq t_{0}(=0)$.

Here $t_{0}$ is the preparation time of the state $\phi$.

The physical meaning of time $t_{0}$ needs some explanation, so that one does not think of $t_{0}$ as a particular time in the life of the experimentalist. Quantum mechanics makes statements about ensembles of microphysical systems. The time $t_{0}$ represents thus an ensemble of times $t_{0}^{1}, t_{0}^{2}, \ldots, t_{0}^{n}, \ldots$ at which the 1 -st, 2 -nd, $\ldots, n$-th, $\ldots$ microphysical system is prepared in the state $\phi^{G}$.

After one has accepted the preparation time $t_{0}$ as the ensemble of times $\left\{t_{0}^{1}, t_{0}^{2}, \ldots, t_{0}^{n}\right\}$ at which the $n$ individual micro-systems have been prepared, we choose this time $t_{0}=\left\{t_{0}^{1}, t_{0}^{2}, \ldots, t_{0}^{n}\right\}$ as the time $t=0$ for the time evolution of a prepared state, e.g. the decaying state vector $\phi^{G}(t)$ of an ensemble of decaying micro-system. The task is then to find a new theory (new boundary conditions replacing Hilbert space axiom (13)). This 
means we need to find new mathematical spaces

for which the solutions of the Schrödinger equation (12c), $\phi(t)=\mathscr{U}^{\times}(t) \phi$, are given by the semigroup $\mathscr{U}^{\times}(t)=\mathrm{e}^{-i H t / \hbar}, 0 \leq t<\infty$

and

for which the solutions of the Heisenberg equation, $\psi(t)=\mathscr{U}(t) \psi$, are given by the semigroup $\mathscr{U}(t)=\mathrm{e}^{i H t / \hbar}, 0 \leq t<\infty$.

\section{The Mathematics of Dirac's Theory}

The first step in that direction has been taken by Schwartz, using for the Dirac formalism of quantum mechanics one Schwartz space $\Phi$

1. The solutions of both the Heisenberg and the Schrödinger equations (for state and observable, respectively) have a Dirac basis vector expansion ${ }^{4}$,

$$
\phi=\sum_{j, j_{3}, \eta} \int d E\left|E, j, j_{3}, \eta\right\rangle\left\langle E, j, j_{3}, \eta \mid \phi\right\rangle,
$$

where the basis vectors $|E\rangle=\left|E, j, j_{3}, \eta\right\rangle$ are "eigenkets" of $H, \mathbf{J}^{2}, J_{3}$ (angular momentum) and other operator $\eta^{\text {op: }}$

$$
\begin{aligned}
& \left\langle\phi|H| E, j, j_{3}, \eta\right\rangle=E\left\langle\phi \mid E, j, j_{3}, \eta\right\rangle \quad \text { for all vectors } \phi \in \Phi . \\
& \mathbf{J}^{2}\left|E, j, j_{3}, \eta\right\rangle=j(j+1)\left|E, j, j_{3}, \eta\right\rangle, J_{3}\left|E, j, j_{3}, \eta\right\rangle=j_{3}\left|E, j, j_{3}, \eta\right\rangle \\
& \text { and } \eta^{\text {op }}\left|E, j, j_{3}, \eta\right\rangle=\eta\left|E, j, j_{3}, \eta\right\rangle
\end{aligned}
$$

To each vector $\phi$ corresponds precisely one function (not a class of functions as for $\left.L^{2}(E)\right)$.

$$
\left\langle E, j, j_{3}, \eta \mid \phi\right\rangle=\phi_{j, j_{3}, \eta}(E)=\langle E \mid \phi\rangle=\phi(E)
$$

2. The co-ordinates or bra-ket $\langle E \mid \phi\rangle=\phi(E)$ are smooth, rapidly decreasing functions of $E$ ("Schwartz function" $\in \mathscr{S}_{\mathbb{R}_{+}}$)

One has a triplet of function spaces and a corresponding triplet of vector spaces

$$
\begin{aligned}
& \{\phi(E)\}=\mathscr{S} \subset L^{2} \subset \mathscr{S}^{\times} \\
& \{\phi\}=\Phi \subset \mathscr{H} \subset \Phi^{\times}
\end{aligned}
$$

The Dirac kets $|E\rangle$ are antilinear continuous Schwartz space functionals $|E\rangle \in \Phi^{\times}$.

The Dirac basis vector expansion (19) is the nuclear spectral theorem in mathematics.

\footnotetext{
${ }^{4} E$ is the continuous analogue of $i$ in $\vec{x}=\sum_{i=1}^{3} \vec{e}_{i} x^{i}$, and $\left\langle E, j, j_{3}, \eta \mid \phi\right\rangle=\phi(E)$ is the continuously infinite analogue of the component $x^{i}=x(i)$.
} 
Thus in Dirac's formulation there is one RHS, and it is the same RHS for the states $\phi$ and the observables $|\psi\rangle\langle\psi|$ :

$$
\{\phi\}=\{\psi\}=\Phi=\text { abstract Schwartz space }
$$

In a theory that distinguishes between prepared states $\{\phi\}$ and detected observables $\{\psi\}$ as indicated by (18S) and (18H) one needs two Rigged Hilbert spaces.

\section{The Mathematics of Time Asymmetric Quantum Mechanics}

To find a time asymmetric quantum theory that incorporates causality as expressed by (17) we start with the

\section{Phenomenological Theory of Scattering and Decay:}

One uses in- and out- plane wave "state" $\left|E^{+}\right\rangle$and $\left|E^{-}\right\rangle$which fulfill the LippmannSchwinger equation [11]

$$
\left|E^{ \pm}\right\rangle=|E \pm i \varepsilon\rangle=|E\rangle+\frac{1}{E-H \pm i \varepsilon} V|E\rangle=\Omega^{ \pm}|E\rangle, \quad \varepsilon \rightarrow+0
$$

One speaks of complex energy:

for the analytic $S$ matrix

for the Gamow states $\phi^{\mathrm{G}}$

$$
\begin{aligned}
& S_{j}(E) \rightarrow S_{j}(z) \\
& z_{R}=E_{R}-i \Gamma / 2
\end{aligned}
$$

for the Lippmann-Schwinger equation or in the propagator of field theory$$
z=E \pm i \varepsilon, \varepsilon \text { infinitesimal }
$$

The $\left|E^{+}\right\rangle$are taken as basis systems for the Dirac basis vector expansion of in-state vectors

$$
\phi^{+}=\sum_{j, j_{3}, \eta} \int_{0}^{\infty} d E\left|E, j, j_{3}, \eta^{+}\right\rangle\left\langle{ }^{+} E, j, j_{3}, \eta \mid \phi^{+}\right\rangle,
$$

and the $\left|E^{-}\right\rangle$are taken as basis systems for the out-vectors

$$
\psi^{-}=\sum_{j, j_{3}, \eta} \int_{0}^{\infty} d E\left|E, j, j_{3}, \eta^{-}\right\rangle\left\langle{ }^{-} E, j, j_{3}, \eta \mid \psi^{-}\right\rangle .
$$

The Dirac basis vector expansions (25), (26) use two different kinds of kets:

$$
\left|E j j_{3} \eta^{\mp}\right\rangle \in \Phi_{ \pm}^{\times} \quad \begin{aligned}
& \text { suggested by the Lippmann-Schwinger out-plane } \\
& \text { waves }\left|E^{-}\right\rangle \text {and in-plane waves }\left|E^{+}\right\rangle, \text {respectively. }
\end{aligned}
$$

Because of the $+i \varepsilon$ in the Lippmann-Schwinger equation, the energy wave function of the prepared in-state $\phi^{+}$:

$$
\phi^{+}(E) \equiv\left\langle{ }^{+} E j j_{3} \eta \mid \phi^{+}\right\rangle=\overline{\left\langle\phi^{+} \mid E j j_{3} \eta^{+}\right\rangle}
$$

is the boundary value of an analytic function in the lower complex energy semi-plane (for complex energy $z=\overline{E+i \varepsilon}=E-i \varepsilon$ immediately below the real axis on the second 
sheet of the $S$-matrix $S_{j}(z)$ ). Similarly, one surmises that the energy wave function of the observable $\left|\psi^{-}\right\rangle\left\langle\psi^{-}\right|$

$$
\psi^{-}(E) \equiv\left\langle^{-} E j j_{3} \eta \mid \psi^{-}\right\rangle
$$

can be extended into an analytic function in the upper complex energy semi-plane. Thus one conjectures:

Two sets of functions

$$
\begin{array}{llll}
\left\{\phi^{+}(E)=\left\langle{ }^{+} E \mid \phi^{+}\right\rangle\right\} & \left\{\phi^{+}\right\} & =\Phi_{-} & \left|E^{+}\right\rangle=\left|E, j, j_{3}, \eta^{+}\right\rangle \\
\left\{\psi^{-}(E)=\left\langle{ }^{-} E \mid \psi^{-}\right\rangle\right\} & \left\{\psi^{-}\right\} & =\Phi_{+} & \left|E^{-}\right\rangle=\left|E, j, j_{3}, \eta^{-}\right\rangle
\end{array}
$$

The spaces $\Phi_{-}$and $\Phi_{+}$will turn out to be well known mathematical spaces, the Hardy spaces (note the change in label for the mathematical spaces and the LippmannSchwinger kets).

From the phenomenological $S$-matrix theory one surmises that the function $\left\langle\psi^{-} \mid E^{-}\right\rangle\left\langle E^{+} \mid \phi^{+}\right\rangle S_{j}(E)$ in the integral of the $S$-matrix element in (29a) and (29b) can be analytically continued into the lower complex semi-plane (second sheet), except for singularities of $S_{j}(E)$; as shown in Fig. 3 .

This means the contour of integration for the $S$-matrix element

$$
\begin{aligned}
\left(\psi^{\text {out }}, S \phi^{\text {in }}\right)= & \left(\psi^{-}, \phi^{+}\right) \\
& =\sum_{j} \int_{E_{0}}^{\infty} d E \sum_{j_{3}} \sum_{\eta, \eta^{\prime}}\left\langle\psi^{-} \mid E, j, j_{3}, \eta^{\prime-}\right\rangle S_{j}^{\eta^{\prime} \eta}(E)\left\langle{ }^{+} E, j, j_{3}, \eta \mid \phi^{+}\right\rangle
\end{aligned}
$$

or in simplified notation:

$$
\left(\psi^{-}, \phi^{+}\right)=\int_{E_{0}}^{\infty} d E\left\langle\psi^{-} \mid E^{-}\right\rangle S_{j}(E){ }^{+} E\left|\phi^{+}\right\rangle
$$

can be deformed, from the positive real axis first sheet $=$ positive real axis second sheet, into the lower complex energy plane second sheet, where the resonance pole of the $S$ matrix is located (e.g. at $z_{R}$ in Fig. 3).

\section{Conjecturing the Hardy Space Axiom}

Therefore the program to determine the property of the spaces $\Phi_{-}$(for states) and $\Phi_{+}$ (for observables) is the following:

Start with pole of $S$-matrix at $z_{R}$ and conjecture the mathematical property of $\left\langle{ }^{-} E \mid \psi^{-}\right\rangle$, $\left\langle{ }^{+} E \mid \psi^{+}\right\rangle$such that a Scattering Resonance and a Decaying State can be derived from the $S$-matrix pole at $z_{R}[12]$.

Consider the simplest case: there is one $S$-matrix pole at $z_{R}=E_{R}-i \Gamma / 2$ on the second sheet, as depicted in Fig. 3. Starting from the integral along the positive real energy axis in (29) one deforms the contour of integration into the second sheet. This leads to the integral along the infinite semicircle $C_{\infty}$ which is zero for any reasonable assumption for the functions $\left\langle\psi^{-} \mid E^{-}\right\rangle=\overline{\psi^{-}(E)}$ and $\left\langle E^{+} \mid \phi^{+}\right\rangle=\phi^{+}(E)$. Then one remains with the 


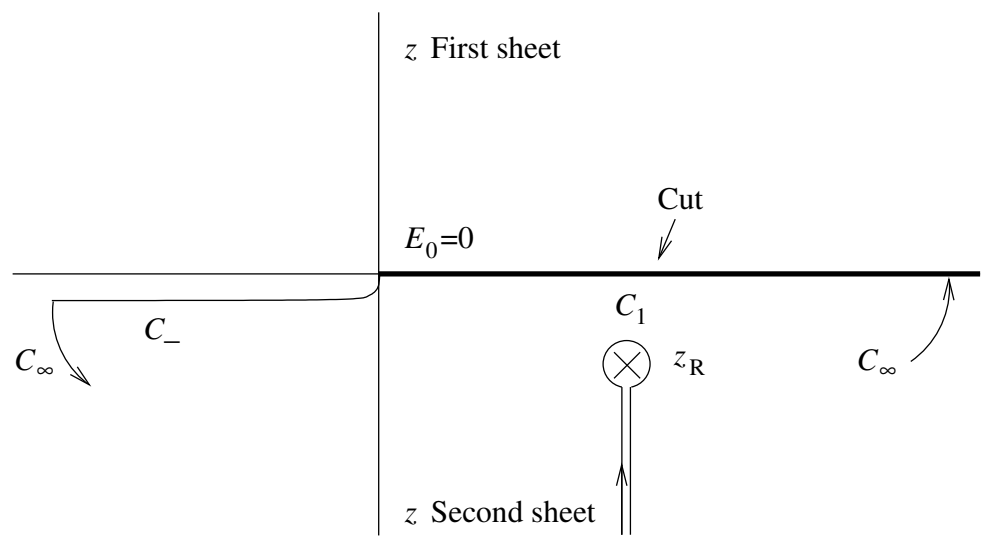

FIGURE 3. The contours of integration for the $S$-matrix element.

integral $C_{-}$in the second sheet, which will describe some background continuum related to $B(E)$ in (6R) (which we shall not discuss here). And there is the integral around the pole position $z_{R}$ (or the sum of these pole terms if there are more than one $S$-matrix pole).

We start with this first order $S$-matrix pole at $z_{R}$ as the definition of a resonance. From the pole term around $z_{R}=E_{R}-i \Gamma / 2$ we derive

1. a Breit-Wigner resonance amplitude

$$
a_{j}^{B W}(E)=\frac{R}{E-z_{R}}, \quad-\infty<E<+\infty
$$

2. a Gamow state vector defined with this Breit-Wigner amplitude

$$
\left|z_{R}, j, j_{3}, \eta^{-}\right\rangle=\int_{-\infty}^{+\infty} d E\left|E, j, j_{3}, \eta^{-}\right\rangle \frac{1}{E-z_{R}}
$$

for which one shows that

1. $\left|z_{R}^{-}\right\rangle$is an eigenket of $H$ with a discrete complex eigenvalue (as Gamow wanted)

$$
H^{\times}\left|E_{R}-i \Gamma / 2^{-}\right\rangle=\left(E_{R}-i \Gamma / 2\right)\left|E_{R}-i \Gamma / 2^{-}\right\rangle
$$

2. it has the property

$$
\begin{aligned}
\left\langle\mathrm{e}^{i H t / \hbar} \psi_{\eta}^{-} \mid E_{R}-i \Gamma / 2^{-}\right\rangle & =\left\langle\psi_{\eta}^{-}\left|\mathrm{e}^{-i H^{\times} t / \hbar}\right| E_{R}-i \Gamma / 2^{-}\right\rangle \\
& =\mathrm{e}^{-i E_{R} t / \hbar} \mathrm{e}^{-(\Gamma / 2) t / \hbar}\left\langle\psi_{\eta}^{-} \mid E_{R}-i \Gamma / 2^{-}\right\rangle,
\end{aligned}
$$

but this property can be derived only for

$$
t \geq t_{0}(=0) \text {. }
$$


The result (31b) shows that the resonance of width $\Gamma$ given by the pole term (30a) is represented by an eigenket (30b) of $H$ with complex eigenvalue (31a), which according to (31b) represents a decaying state with lifetime $\tau=\frac{\hbar}{\Gamma}$.

Summarizing: the pole of the $S$-matrix can be mathematically represented by a BreitWigner amplitude (30a) and by a Gamow vector (31b) which is an exponentially decaying state $(31 \mathrm{~b})$ with a lifetime:

$$
\tau=\frac{\hbar}{\Gamma}
$$

In order to derive these results (30) and (31), specific mathematical assumptions must be made about the mathematical properties of energy wave functions $\left\langle{ }^{+} z \mid \phi^{+}\right\rangle$and $\left\langle{ }^{-} z \mid \psi^{-}\right\rangle$that appear in the integral (29) for the $S$-matrix element. These conditions lead to the new hypothesis [12]:

The energy wave functions of a state are smooth Hardy functions analytic on the lower complex plane $\mathbb{C}_{-}$:

$$
\phi^{+}(E)=\left\langle{ }^{+} E \mid \phi^{+}\right\rangle \in\left(\mathscr{H}_{-}^{2} \cap \mathscr{S}\right)_{\mathbb{R}_{+}}=\text {Hardy functions } \mathbb{C}_{-} .
$$

The energy wave functions of an observable are smooth Hardy functions analytic on the upper complex plane $\mathbb{C}_{+} .{ }^{5}$

$$
\psi^{-}(E)=\left\langle^{-} E \mid \psi^{-}\right\rangle \in\left(\mathscr{H}_{+}^{2} \cap \mathscr{S}\right)_{\mathbb{R}_{+}}=\text {Hardy functions } \mathbb{C}_{+},
$$

where $\mathbb{C}_{-}\left(\mathbb{C}_{+}\right)$denotes the lower (upper) complex energy semi-plane of the $S$-matrix $S_{j}(E)$ in (29).

Therewith we have inferred from heuristic results a new mathematical axiom of causal quantum theory which applies to resonances and decay:

\section{the Hardy space axiom}

The set of prepared (in-) states defined by

the preparation apparatus (e.g., accelerator) is $\left\{\phi^{+}\right\}=\Phi_{-} \subset \mathscr{H} \subset \Phi_{-}^{\times}$

The set of (out-) observables defined by the registration apparatus (e.g. detector)

is $\quad\left\{\psi^{-}\right\}=\Phi_{+} \subset \mathscr{H} \subset \Phi_{+}^{\times}\left(34_{+}\right)$ where $\Phi_{\mp}$ are Hardy spaces of the semiplanes $\mathbb{C}_{\mp}$ and the spaces $\Phi_{\mp}^{\times}$are the duals (i.e., the spaces of antilinear continuous Hardy space functionals).

This new axiom gives a mathematical meaning to Lippmann-Schwinger kets $\left|E^{ \pm}\right\rangle$and to the Gamow kets $\left|E_{R}-i \Gamma / 2^{-}\right\rangle$as functionals on the Hardy spaces $\Phi_{\mp}$. And it provides a consistent mathematical theory which unifies resonance and decay phenomena, and the resonance state vector (30b) obeys the exact exponential decay law (31b).

Accepting the new Hardy space axiom $\left(33_{ \pm}\right)$or $\left(34_{ \pm}\right)$, the dynamical equations (12ac) are now solved under the Hardy space boundary conditions (34 \pm ) and not the Hilbert space boundary condition like in (13a). From the Hardy space axiom does not follow the unitary group evolution (14) which was a consequence of the Stone-von Neumann theorem of the Hilbert space. But in Hardy space a similar theorem holds, the PaleyWiener theorem. By the Paley-Wiener theorem the solution of the same dynamical equations (12a), (12b), (12c) are given instead by the semigroup solutions (18S) for the state

\footnotetext{
5 This is because their complex conjugates $\overline{\psi^{-}(E)}=\left\langle\psi^{-} \mid E^{-}\right\rangle$are Hardy on $\mathbb{C}_{-}$.
} 
vector $\phi^{+}(t)$ and $(18 \mathrm{H})$ for the observable vector $\psi^{-}(t)$. This means the mathematical consequence of the Hardy space axiom $\left(33_{ \pm}\right)$agrees with the phenomenological condition (17) expressing causality and avoiding the "exponential catastrophe" (15).

The property $t \geq 0$ in (31b) is thus also a mathematical consequence of the Hardy space axiom $\left(33_{+}\right)$for the Gamow ket, $\left|z_{R}, j, j_{3}, \eta^{-}\right\rangle \in \Phi_{+}^{\times 6}$.

From the result (31b) follows that a resonance of width $\Gamma$ (30a) is an exponentially decaying state with lifetime $\tau=\hbar / \Gamma$; this is an exact relation.

If one observes in experiments "deviations from the exponential law," then it is due to the fact that one has measured not the time evolution of the Gamow state $\phi_{j}^{G}=\left|E_{R}-i \Gamma / 2^{-}\right\rangle$of (30), (31a), (31b), but the time evolution of a superposition (or of a mixture), $\phi_{j}^{G}+\phi_{j}^{B}$, of $\phi_{j}^{G}$ the exponentially decaying state and some background $\phi_{j}^{B}$, where $\phi_{j}^{B}$ is connected with a background amplitude $B(E)$ in (6R), which theoretically is contained in the integral along $C_{-}$in Fig. 3. The exponential time dependence displayed in Fig. 2 has been obtained in [6] only after the $K_{L}^{0}$ interference and other contributions have been eliminated from the fit in Fig. 2.

The Gamow ket $\left|z_{R}^{-}\right\rangle$, like the Dirac ket, is a mathematical representation of an idealized state. With a macroscopic apparatus one cannot detect a single energy value out of an energy continuum; this does not diminish the importance of these "singular" mathematical objects, like Dirac kets or Gamow kets, for the understanding of quantum physics.

\section{THE EXPONENTIAL DECAY OF RELATIVISTIC RESONANCES AND THE DEFINITION OF Z-BOSON MASS}

For the relativistic resonance, specifically the $Z$-boson, one has a multitude of parametrizations of which those of equations (1)-(5) are popular ones. The $j^{\text {th }}$ partial scattering amplitude in a relativistic resonance formation process $a_{j}(s)$ of (4) is a function of the invariant mass square $s=\left(p_{1}^{\mu}+p_{2}^{\mu}\right)^{2}=\left(E_{1}^{\mathrm{cm}}+E_{2}^{\mathrm{cm}}\right)^{2}$, where $p_{1}^{\mu}, p_{2}^{\mu}$ are the momenta of the two incoming (or outgoing) particles. One desires to write the amplitude of a resonance scattering process as the sum (4), where $B_{j}(s)$ is the nonresonant background and $a_{j}^{\text {res }}$ describes the resonance per se. It is not possible to fix the functions $a_{j}^{\text {res }}(s)$ and $B_{j}(s)$ separately using only the experimental cross section data for $\left|a_{j}(s)\right|^{2}$. One needs some theoretical arguments in favor of a particular functional form of $a_{j}^{\text {res }}$, like, e.g., the Breit-Wigner form (3), or e.g. the on-mass-shell definitions (1a), or the parametrization (2), since only $\left|a_{j}(s)\right|^{2}$ is experimentally available in scattering experiments, and not $a_{j}^{\text {res }}(s)$ and $B_{j}(s)$ separately.

\footnotetext{
${ }^{6}$ The miss-match in the notation for labels + and - for the kets $\left|z_{R}^{-}\right\rangle$and spaces $\Phi_{+}^{\times}$has its origin in the physicists notation $\left|E^{\mp}\right\rangle$ for the Lippmann-Schwinger kets and the mathematicians notation for the Hardy function spaces $\mathscr{H}_{ \pm}$and thus for the abstract spaces: $\Phi_{ \pm} \ni\left|E_{\mp}\right\rangle$. Except for the miss-match in the notation \pm , the correspondence between the mathematics of Hardy spaces and the physics of quantum and decay phenomena is a beautiful example of what Wigner called "the miracle of appropriateness of the language of mathematics for the formulation of the laws of physics", E.P. Wigner, Symmetries and Reflections, Ch. 17 Ox Bow Press, Woodbridge, Connecticut, 1979.
} 
This means that the selection of the "right" $(M, \Gamma)$ requires a theory that relates $M$ or $\Gamma$ to the particle aspects of the relativistic decaying state.

Relativistic particles, stable or decaying, are by hypotheses given by the representations of the inhomogeneous Lorentz transformations [13], as is the standard for stable relativistic particles [5]. Therefore:

Stable relativistic particles are represented by unitary representations of Poincaré group with (mass,spin) given by

$$
(m, j)=(\text { real }, \text { semi-integer })
$$

Decaying relativistic particles we represent by semigroup representations of (complex mass, spin $)=\left(\sqrt{s_{R}}, j\right)$

$$
\sqrt{s_{R}}=\left(M_{R}-i \frac{\Gamma_{R}}{2}\right)=\sqrt{\bar{M}_{Z}^{2}-i \bar{M}_{Z} \bar{\Gamma}_{Z}} .
$$

The basis vectors of the semigroup representation $\left[s_{R}, j\right]$ are given, in very close analogy to the non-relativistic Gamow vectors (30b) by

$$
\left|\left[j, s_{R}\right], \hat{\mathbf{p}}, j_{3}^{-}\right\rangle=\frac{1}{2 \pi} \int_{-\infty I I}^{\infty} d s\left|[j, s], \hat{\mathbf{p}}, j_{3}^{-}\right\rangle \frac{1}{s-s_{R}},
$$

where

$$
\hat{\mathbf{p}}=\frac{\mathbf{p}}{S} \equiv \gamma \mathbf{v} ; \quad \hat{p}^{0} \equiv \gamma=\left(\sqrt{1-\mathbf{v}^{2}}\right)^{-1}
$$

are the components of the 4-velocity.

The Gamow vectors (36) have been defined in complete analogy to the non-relativistic Gamow vectors (30b) except that in place of $E$, the relativistically invariant energy square $s$ has been used. In the same way as for the non-relativistic case (31a) one can show that the $\left|\left[j, s_{R}\right], \hat{\mathbf{p}}, j_{3}^{-}\right\rangle$are generalized eigenvectors of the total invariant mass square operator $P^{\mu} P_{\mu}$

$$
P^{\mu} P_{\mu}\left|\left[j, s_{R}\right], \hat{\mathbf{p}}, j_{3}^{-}\right\rangle=s_{R}\left|\left[j, s_{R}\right], \hat{\mathbf{p}}, j_{3}^{-}\right\rangle .
$$

The value $s_{R}$ in (36) is the position of the resonance pole of the analytically continued $S$-matrix, the relativistic analogue of $z_{R}$ in Fig. 3, which is on the second sheet of the Riemann surface of the $S$-matrix element $S_{j}(s)$. The integration in (36) extends over the real energy axis on the second sheet, which coincides with the physical values on the lower edge of the first sheet for $s \geq m_{0}^{2}$. This is as shown in Fig. 3, except that the complex energy $z$ has to be replaced by the complex invariant mass squared $s=p^{\mu} p_{\mu}=\left(p_{1}+p_{2}\right)^{\mu}\left(p_{1}+p_{2}\right)_{\mu}$.

As mentioned in the Introduction, for the relativistic resonance, specifically for the $Z$-boson, one has a multitude of parametrizations of which those of Eqs. (1)- (3) are the popular ones. Fitting $\left|a_{j}(s)\right|^{2}$ of (4) to the experimental data, like in Fig. 1, cannot discriminate between the various choices (1a), (2) and (3), etc., because one can shift the difference between, e.g., (1a) and (3) into the unknown background $B_{j}(s)$ of (4). The selection of the "right" parameters $(M, \Gamma)$, i.e., the choice of the amplitude, which one associates to the resonance per se, requires a theory that relates $M$ and $\Gamma$ to the particle aspect of the relativistic decaying state. This is given by the irreducible representations of the inhomogeneous Lorentz transformations [13], as a generalization of Wigner's choice of the unitary representations for stable relativistic particles [5]. 
To calculate the time evolution of these basis vectors (36) for the semigroup representation one proceeds in the following way:

Go to the rest frame $\hat{\mathbf{p}}=(1,0,0,0), \mathbf{v}=0$. Then (37) becomes:

$$
H^{\times}\left|\left[j, s_{R}\right], \hat{\mathbf{p}}=0, j_{3}^{-}\right\rangle=\sqrt{s_{R}}\left|\left[j, s_{R}\right], \hat{\mathbf{p}}=0, j_{3}^{-}\right\rangle, \quad H^{\times}=P_{0} .
$$

The relativistic state at time $t$ is then obtained by the time evolution in the rest frame; $(\hbar=1)$ :

$$
\begin{aligned}
\psi_{s_{R}}^{G}(t)=\mathrm{e}^{-i H^{\times}}\left|\left[s_{R}, j\right] \hat{\mathbf{p}}=0, j_{3}^{-}\right\rangle= & \mathrm{e}^{-i \sqrt{s_{R}} t}\left|\left[s_{R}, j\right] \hat{\mathbf{p}}=0, j_{3}^{-}\right\rangle \\
& =\mathrm{e}^{-i M_{R} t} \mathrm{e}^{-\left(\Gamma_{R} / 2\right) t}\left|\left[s_{R}, j\right] \hat{\mathbf{p}}=0, j_{3}^{-}\right\rangle, \quad t \geq 0 .
\end{aligned}
$$

From this one obtains, for instance, that the "survival probability at a time $t \geq 0$ is proportional to

$$
\left|\left\langle\psi^{G} \mid \psi_{s_{R}, j}^{G}(t)\right\rangle\right|^{2}=\mathrm{e}^{-\Gamma_{R} t}\left|\left\langle\psi^{G} \mid \psi_{s_{R}, j}^{G}(0)\right\rangle\right|^{2}, \quad t \geq 0 \quad \text { only. }
$$

This means that the lifetime $\tau$ of the exponential decay is given by

$$
\tau=\frac{1}{\Gamma_{R}} .
$$

Only $\Gamma_{R}$ of (3), and not $\bar{\Gamma}_{Z}$, nor $\Gamma_{Z}$ of (1a) will be the inverse of an exponential lifetime.

One can start with the definition of the (first order) resonance by the pole of the $j$-th partial $S$-matrix element $S_{j}(s)$, cf., (29) and obtain the relativistic Gamow vector in analogy to the non-relativistic case (30b) as the vector (36) with "relativistic BreitWigner" line shape $\frac{1}{s-s_{R}}$ (obtained from the integral around circle $C_{1}$ of the pole at $s_{R}$ by contour deformation). Then one obtains the exponential time evolution (38) and (39) which is the relativistic version of (31b) - the lifetime for the Gamow vector (36) is then derived as (40).

The semigroup time evolutions, $t \geq 0$ in (38) for the relativistic Gamow states at rest $\left|\left[j, s_{R}\right], \hat{\mathbf{p}}=0, j_{3}^{-}\right\rangle$are just the subset of the causal transformations of the relativistic space time. The set of all causal transformations is given by the Poincaré semigroup into the forward light cone,

$$
\mathscr{P}_{+}=\left\{(\Lambda, x): x^{2}=t^{2}-\mathbf{x}^{2} \geq 0, t \geq 0\right\} .
$$

To summarize, of the many possible choices for the definition of mass $M$ for a relativistic resonance, of which $\left(M_{Z}, \Gamma_{Z}\right),\left(\bar{M}_{Z}, \bar{\Gamma}_{Z}\right)$ and $\left(M_{R}, \Gamma_{R}\right)$ in (1)- (3) are some examples, only the parametrization $s_{R}=\left(M_{R}-i \Gamma_{R}\right)^{2}$ for the pole position of the $S$-matrix pole, unifies the various concepts and heuristic notions that have been used successfully in the phenomenological and mathematical description of relativistic resonances and relativistic decaying states: it unifies the Breit-Wigner resonance (36) with lineshape (3) and the exponentially decaying Gamow state (36) with (39), (40). And it relates both to the resonance pole of the $S$-matrix. The relativistic version of causal time evolution (38), (39) is a special case of "Einstein causality" (41), which states that causal symmetry 
transformations of relativistic space time are given by the Poincaré semigroup transformation (41).

The lifetime-width relation

$$
\tau=\frac{\hbar}{\Gamma}
$$

is an exact relation, in the non-relativistic as well as in the relativistic case.

All this is accomplished by a single modification of one mathematical axiom of quantum theory. Instead of choosing for the energy functions (21) the Hilbert space or the Schwartz space wave functions (22), one needs to use the Hardy space axiom $\left(34_{ \pm}\right)$and choose the analytic Hardy wave functions, which distinguish mathematically between states (34_) and observables (34+).

The Hardy space theory can be applied specifically to the question: What should be used for the mass and the width of the Z-boson, and similarly for the mass and width of any relativistic resonance? The theory, which is based on not much more than the condition of causality, which stated that the Born probabilities (17) need to exist only after the state was prepared, suggests that $\left(M_{R}, \Gamma_{R}\right)$ is the right definition of the mass and width for a relativistic resonance. Specifically for the $Z$-boson it means that

$$
\begin{aligned}
M_{R} & =\operatorname{Re} \sqrt{s_{R}}=91.1611 \pm 0.0023 \mathrm{GeV}=M_{Z}-0.026 \mathrm{GeV} \\
\Gamma_{R} & =-2 \operatorname{Im} \sqrt{s_{R}}=2.4943 \pm 0.0024 \mathrm{GeV}
\end{aligned}
$$

are the right values of mass and width of the $Z$-boson, not $\left(\bar{M}_{Z}, \bar{\Gamma}_{Z}\right)$ or $\left(M_{Z}, \Gamma_{Z}\right)$.

It is therefore difficult to appreciate that the more recent editions of the particle data table [3] quote exclusively the values for $\left(M_{Z}, \Gamma_{Z}\right)$ of (1a), which is specific to the onthe-mass-shell-renormalization scheme, especially since it is known now that this choice is not gauge invariant.

These were precisely the questions that concerned Augusto in his scientific work. Starting with his detailed calculations for the hyperon semileptonic decay data in his dissertation at the University of Chicago, in close collaboration with the experimental colleagues, his principal interest was to bring experiment and theory together. Therefore it was quite natural, that he was agitated about experimental masses, which violated the theoretical principles of gauge invariance, and stirred me up about it.

Augusto has been for many years a valuable collaborator and a dear friend and adviser, not only on professional matters, but also on personal problems; wise, intelligent and helpful, he was a joy to be with. A passionate music lover, particularly of Richard Strauss and Mozart, Augusto with his authoritative bass voice, could discuss the intricacies of Chicago politics and yet, within the next sentence, reveal his depth of understanding of the human condition.

\section{ACKNOWLEDGMENTS}

The author would like to thank Miguel A. Perez and the administration of CINVESTAV for providing this opportunity to express our appreciation of Augusto Garcia for his contributions to physics and to the enrichment of our lives. 
He expresses his gratitude to P. Kielanowski and P. Bryant for their help with this manuscript. He also gratefully acknowledges partial support by the US NSF (OISE0421936).

\section{REFERENCES}

1. S. Eidelman et al. (Particle Data Group), Phys. Lett. B592, 1 (2004).

2. A. Sirlin, Phys. Rev. Lett. 67, 2127-2130 (1991); A. Sirlin, Phys. Lett. B267, 240-242 (1991); S. Willenbrock, and G. Valencia, Phys. Lett. B259, 373-376 (1991); R. Stuart, Phys. Lett. B262, 113-119 (1991); R. Stuart, Phys. Lett. B272, 353-358 (1991); A. Leike, T. Riemann, and J. Rose, Phys. Lett. B273, 513-518 (1991).

3. C. Amsler et al. (Particle Data Group), Phys. Lett. B667, 1 (2008).

4. A. Bernicha, G. López Castro, and J. Pestieau, Nucl. Phys. A 597, 623-635 (1996); A. Bernicha, G. López Castro, and J. Pestieau, Phys. Rev. D 50, 4454-4461 (1994).

5. S. Weinberg, The Quantum Theory of Fields, vol. I Foundations, Cambridge University Press, 1995.

6. L. K. Gibbons et al., Phys. Rev. D 55, 6625-6715 (1997); L. K. Gibbons, A precise measurement of the $C P$ violation parameter $\operatorname{Re}\left(\varepsilon^{\prime} / \varepsilon\right)$ and other kaon decay parameters, Ph.D. thesis, Univ. of Chicago (1993); L. K. Gibbons et al., Phys. Rev. Lett. 70, 1199-1202 (1993).

7. V. Weisskopf, and E. Wigner, Z. Phys. 63, 54 (1930); V. Weisskopf, and E. Wigner, Z. Phys. 65, 18 (1930).

8. M. Goldberger, and K. Watson, Collision Theory, Wiley, New York, 1964, chap. 8.

9. G. Gamow, Z. Phys. 51, 204 (1928); cf. also J.J. Thomson, Proc. London Math. Soc. 15, 197 (1884).

10. J. von Neumann, Mathematical Foundations of Quantum Mechanics, Springer Verlag, Berlin, 1932, Princeton University Press, 1995.

11. B. Lippmann, and J. Schwinger, Phys. Rev. 79, 469 (1950); M. Gell-Mann, and H. Goldberger, Phys. Rev. 91, 398 (1953); R. Haag, Fortschr. Phys. 7, 183 (1959); R. Newton, Scattering Theory of Waves and Particles, Springer-Verlag, New York, 1982, 2nd ed., chap. 7.

12. For the non-relativistic case this derivation is given, e.g., in A. Bohm, Quantum Mechanics: Foundations and Applications, 3-rd Edition, Springer-Verlag, New York (1979), Section XXI.4.

13. For the relativistic case see A. Bohm, H. Kaldass, S. Wickramasekara, Fortschr. Phys. 51, No. 6 (2003). 